\title{
The Influence of the Magnetic Field on the Dynamics of the Flux Jumps in the Flux Flow Model
}

\author{
S. VAsiliev ${ }^{a}$, A. NAbialek ${ }^{b, *}$, V.F. Rusakov ${ }^{c}$, L.V. Belevtsov ${ }^{a}$, \\ V.V. Chabanenko ${ }^{a}$ And H. SzYMCZaK ${ }^{b}$ \\ ${ }^{a}$ Institute for Physics and Engineering, NASU, 72 ul. R. Luxemburg, 83114, Donetsk, Ukraine \\ ${ }^{b}$ Institute of Physics, Polish Academy of Sciences, al. Lotników 32/46, 02-668 Warsaw, Poland \\ ${ }^{\mathrm{c}}$ National University, 24 Universitetskaya str., 83055, Donetsk, Ukraine
}

\begin{abstract}
We studied the dynamics of the flux jumps in type II superconductors assuming this dynamics to be determined by magnetic diffusion. The conductivity of the sample during the jump was described by the flux flow conductivity, which depends on local magnetic field. We solved this problem numerically for an infinite slab sample. We assumed that superconducting sample was fully penetrated by the magnetic flux at the beginning and that the local induction of the magnetic field, in the whole sample, was equal to $B_{0}$. Then, the external magnetic field was increased by a value of $\Delta B_{\mathrm{a}}$ and in the external magnetic field of $B_{0}+\Delta B_{\mathrm{a}}$ flux jump occurred. In our simulations we used the parameters characteristic of a conventional NbTi superconductor at $4.2 \mathrm{~K}$. In particular, we studied the influence of the parameter $B_{0}$ on the flux jumps' dynamics. We have found that this parameter strongly influences initial stage of the diffusion process. With increasing $B_{0}$, the time during which flux front of the avalanche reaches the center of the sample decreases. The results of numerical simulations were compared with experiments.
\end{abstract}

PACS numbers: 74.25.Sv, 74.70.Ad

\section{Introduction}

At certain conditions, critical state of superconducting sample may become unstable leading to a thermomagnetic avalanche called flux jump. These avalanches are challenging from the viewpoint of superconductors' applications. Hence, it is important to study both the conditions of the avalanche development and the dynamics of this process [1].

The dynamics of the thermomagnetic avalanches was studied by many authors, however it remains still poorly understood. In Ref. [2], the authors determined the limits of application of the Bean model in such studies, assuming the critical current density to be constant in the whole sample volume. With the increase of temperature, they found an increase of the magnetic diffusion caused by an increase of resistivity. The influence of the voltage-current characteristic on the magnetic flux dynamics was considered in Ref. [3]. It was shown that the Mawstari model [4], assuming the critical current density to change in the sample volume, describes the thermomagnetic avalanche better than the Bean model in the case of a flux creep regime as well as in the case when the voltage-current characteristic of the superconductor is described by a step-like function.

It is also important to understand the propagation of an impulse of the external magnetic field in a volume of a high temperature superconductor, which can be described as a diffusion process. Such investigations for the

* corresponding author; e-mail: nabia@ifpan.edu.pl flux-flow regime were performed in Ref. [5]. As the flux creep occurs only for a very low electric field (lower than about $\left.1 \mu \mathrm{V} \mathrm{cm}^{-1}[6]\right)$, the voltage-current characteristic can be approximated by the function

$$
E= \begin{cases}\rho_{\mathrm{f}}\left(j-j_{\mathrm{c}}\right), & E>0, \\ \rho_{\mathrm{f}}\left(j+j_{\mathrm{c}}\right), & E<0,\end{cases}
$$

where $\rho_{\mathrm{f}}$ is the flux flow resistivity and $j_{\mathrm{c}}$ is the critical current density. It was shown in Ref. [1] that the dynamics of the flux jump practically does not depend on thermal properties of the investigated sample. However, in a more precise analysis, it is necessary to take into account the temperature dependence of $\rho_{\mathrm{f}}$ and $j_{\mathrm{c}}$. Recently, the dynamics of the thermomagnetic avalanches in the form of dendrites, which occur in superconducting thin films, was intensively studied $[7,8]$.

In our present model, we assumed that the dynamics of the magnetic flux jump is determined by magnetic diffusion. We considered an infinite slab sample and the resistivity of the sample described by the flux flow resistivity model. In particular, we study the influence of the magnetic field on the dynamics of the flux jumps in such model. The problem was solved numerically. In our simulations, we used the parameters characteristic of conventional $\mathrm{NbTi}$ superconductor. The aim of the present work is to compare the predictions of numerical simulation with experimental data.

\section{Method}

Assuming flux flow conductivity $\sigma_{\mathrm{ff}}=\sigma_{\mathrm{n}} B_{\mathrm{c} 2} / B$, where $\sigma_{\mathrm{n}}$ is the normal state conductivity, $B_{\mathrm{c} 2}$ is the induction 
of the second critical field, and $B$ is the induction of the local magnetic field, we solved numerically the following equation of magnetic diffusion:

$$
\frac{\partial B}{\partial t}=\frac{1}{\mu_{0} \sigma_{\mathrm{n}} B_{\mathrm{c} 2}} \frac{\partial}{\partial x}\left(B \frac{\partial B}{\partial x}\right),
$$

with appropriate initial and boundary conditions. We assumed that at the beginning, the superconducting sample was fully penetrated by the magnetic flux and that the local induction of the magnetic field was equal to $B_{0}$ in the whole sample. Then, the external magnetic field was slowly increased by a value of $\Delta B_{\mathrm{a}}$ inducing in the superconducting slab critical state with the screening current density of $j_{\mathrm{c}}$. In the external magnetic field of $B_{0}+\Delta B_{\mathrm{a}}$, we assumed a flux jump to occur. Hence, in order to calculate the dynamics of the flux jump, we took initial condition (see also Fig. 1 and Fig. 2 with the magnetic field distribution for $t=0)$ :

$$
\begin{aligned}
& B(x, 0)=B_{0}+\left[\Delta B_{\mathrm{a}}-\mu_{0} j_{\mathrm{c}}(d-|x|)\right] \\
& \quad \times \theta\left[\Delta B_{\mathrm{a}}-\mu_{0} j_{\mathrm{c}}(d-|x|)\right],
\end{aligned}
$$

where $\theta$ is the Heaviside step function, and the boundary conditions

$$
B( \pm d, t)=B_{0}+\Delta B_{\mathrm{a}} .
$$

We applied in our studies an implicit method of solving partial differential equations. We performed our simulations for various $B_{0}, d=3 \mathrm{~mm}$ and other parameters characteristic of conventional NbTi superconductor: $j_{\mathrm{c}}=10^{9} \mathrm{~A} / \mathrm{m}^{2}, B_{\mathrm{c} 2}=17 \mathrm{~T}, \sigma_{\mathrm{n}}=4.17 \times 10^{6} \Omega^{-1} \mathrm{~m}^{-1}$ and $\Delta B_{\mathrm{a}}=0.3 \mathrm{~T}$

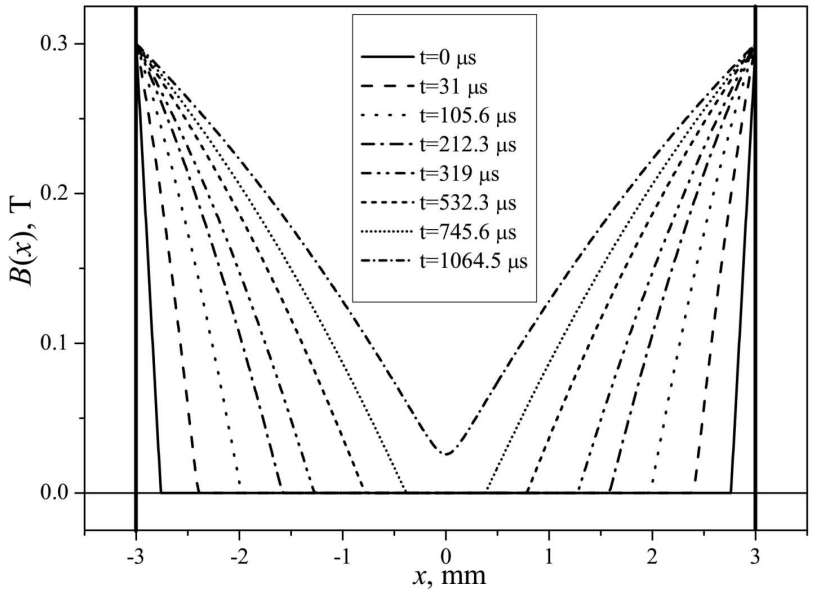

Fig. 1. Time evolution of magnetic field profiles in an infinite slab with thickness $2 d=6 \mathrm{~mm}$, for $B_{0}=0$. The evolution during a period of about $1 \mathrm{~ms}$, is shown.

\section{Results}

Figure 1 shows time evolution of magnetic field profiles in our sample for $B_{0}=0$. The figure shows the evolution during a period of about $1 \mathrm{~ms}$, when the front of the magnetic flux reaches the center of the sample. Figure 2

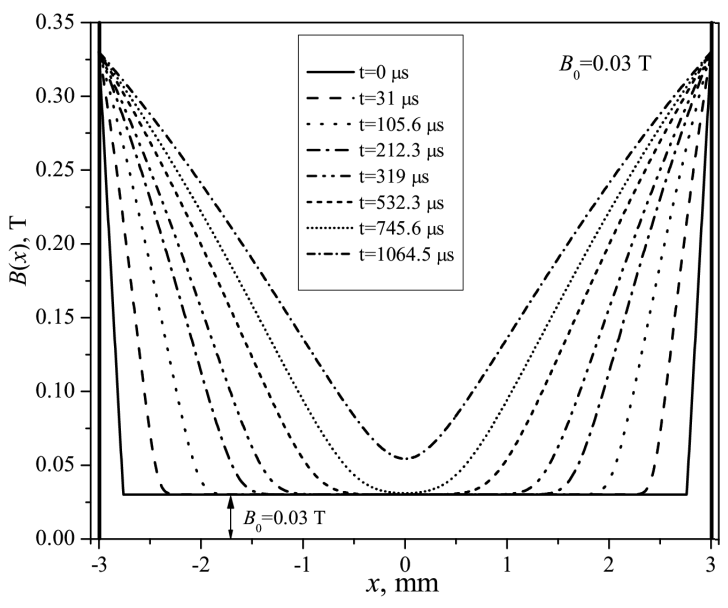

Fig. 2. Time evolution of magnetic field profiles in an infinite slab with thickness $2 d=6 \mathrm{~mm}$, for $B_{0}=0.03 \mathrm{~T}$. The evolution during a period of about $1 \mathrm{~ms}$, is shown.

shows similar evolution for $B_{0}=0.03 \mathrm{~T}$. It is clearly seen that even such small initial magnetic field changes significantly the magnetic field profiles at the initial stage of the process analyzed in our simulations.

Figure 3 presents the calculated time dependence of the magnetic field in the center of the slab for different values of $B_{0}$ (from 0 to $0.07 \mathrm{~T}$ ). The duration of the flux jumps is of several milliseconds. One can find that with the increase of $B_{0}$, the front of the magnetic flux reaches the center of the sample more rapidly. However, the influence of the initial magnetic field on the duration of the total flux jump is relatively weak.

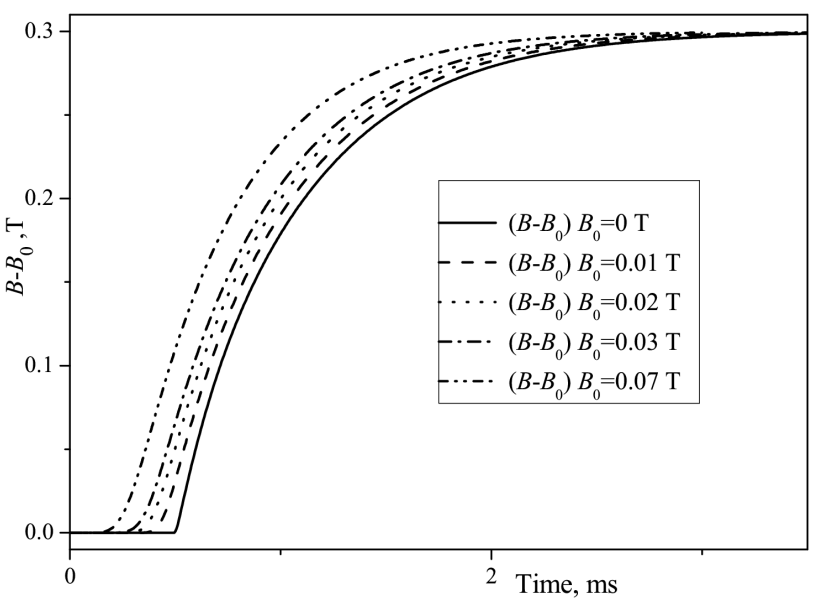

Fig. 3. Time dependence of the magnetic field in the center of the slab, for different values of $B_{0}$.

The time after which induction in the sample center increases by $1 \%$ of $\Delta B_{\mathrm{a}}$ as a function of $B_{0}$ is shown, in logarithmic scale, in Fig. 4. This time decreases with the increase of $B_{0}$, most rapidly for smallest values of $B_{0}$. 


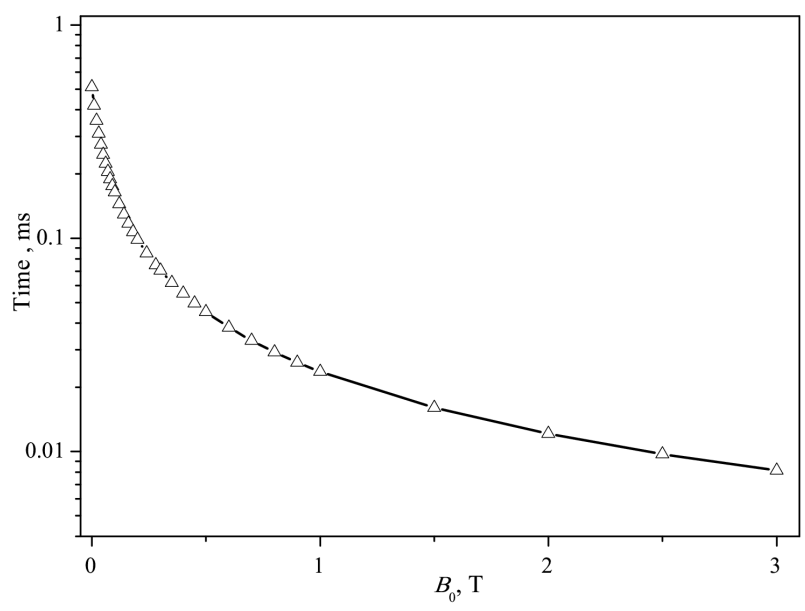

Fig. 4. Time after which induction in the sample center increases by $1 \%$ of $\Delta B_{\mathrm{a}}$ as a function of $B_{0}$.

\section{Discussion}

In our experimental works $[9,10]$ we studied the dynamics of flux jumps in NbTi disk and cylinder. We analyzed the duration of the following flux jumps as a function of the external magnetic field. In superconducting disk, flux jumps occurred in the external magnetic field range about $\pm 3 \mathrm{~T}$. The duration of the following flux jumps was of several milliseconds and their magnetic field dependence was relatively weak. In Ref. [9] we also analyzed two characteristic stages of the flux jumps, they were: (1) first stage, when the rate of the magnetic flux changes increases, and (2) second stage, when the rate of the magnetic flux changes decreases. Magnetic field dependence of the duration of these two stages was slightly different. A more strong dependence was observed in the case of the first stage. However, the experimentally observed magnetic field dependence of the first stage duration (maximal changes by a factor of about 5) was not so strong as we could expect from the present simulation (two orders of magnitude, see Fig. 4). The experimentally observed changes of the duration of the first stage of the avalanche were also not so systematic as those predicted by numerical simulations.

It is, however, important to emphasize that the initial stage of the thermomagnetic avalanche with the increasing rate of the magnetic flux changes cannot be considered in the terms of the magnetic diffusion, because for the magnetic diffusion processes we always expect the rate of the magnetic flux changes to be reduced with time. In an appropriate analysis of the initial stage of the thermomagnetic avalanche it is necessary to take into account a positive feedback between magnetic and thermal fluctuations in the superconducting sample [11]. On the other hand, the second stage of the thermomagnetic avalanche with the reducing rate of the magnetic flux changes, has typical diffusive character.

\section{Conclusions}

If one describes the dynamics of the flux jump as a magnetic diffusion with the diffusivity proportional to the flux flow resistivity, one can expect an influence of the initial magnetic field, frozen in the sample before the flux jump, on the dynamics of flux jumps. This influence is more significant at the initial stage of the diffusion process. With increasing initial magnetic field, the time period decreases when the flux front reaches the center of the sample.

However, at the initial stage of the thermomagnetic instability the model of the magnetic diffusion is not sufficient to describe correctly dynamics of the experimentally observed flux jumps. On the other hand, the final stage of the experimentally observed avalanches has typical diffusive character.

\section{Acknowledgments}

This work was partly supported by Polish Ministry of Science and Higher Education under research project for years 2007-2009 (grant N N202 1285 33).

\section{References}

[1] M.R. Wertheimer, J. le G. Gilchrist, J. Phys. Chem. Solids 28, 2509 (1967).

[2] Y.S. Cha, Physica C 330, 1 (2000).

[3] T. Sueyoshi, T. Fujiyoshi, K. Miyahara, S. Awaji, Supercond. Sci. Technol. 12, 55 (1999).

[4] Y. Mawstari, A. Sawa, H. Obara, M. Umeda, H. Yamasaki, Appl. Phys. Lett. 70, 2300 (1997).

[5] V. Meerovich, M. Sinder, V. Sokolovsky, S. Goren, G. Jung, G.E. Shter, G.S. Grader, Supercond. Sci. Technol. 9, 1042 (1996).

[6] M. Polak, P. Koffman, M. Majoros, M. Kedrova, V. Plechacek, Supercond. Sci. Technol. 3, 67 (1990).

[7] I. Aranson, A. Gurevich, V. Vinokur, Phys. Rev. Lett. 87, 067003 (2001).

[8] I.S. Aranson, A. Gurevich, M.S. Welling, R.J. Wijngaarden, V.K. Vlasko-Vlasov, V.M. Vinokur, U. Welp, Phys. Rev. Lett. 94, 037002 (2005).

[9] S. Vasiliev, A. Nabiałek, V. Chabanenko, V. Rusakov, S. Piechota, H. Szymczak, Acta Phys. Pol. A 109, 661 (2006).

[10] A. Nabiałek, S. Vasiliev, V. Chabanenko, V. Rusakov, S. Piechota, H. Szymczak, Acta Phys. Pol. A 114, 235 (2008).

[11] R.G. Mints, A.L. Rakhmanov, Rev. Mod. Phys. 53, 551 (1981). 